\title{
SYN-1,4-ADDITION OF CARBOXYLATE SALTS TO CYCLIC ALLYLIC EPOXIDES MEDIATED BY CUPROUS CHLORIDE
}

\author{
J. P. Marino* and Juan C. Jaén \\ Department of Chemistry, The University of Michigan, \\ Ann Arbor, Michigan 48109
}

Summary: A mild method for the 1,4-syn opening of certain cyclic allylic epoxides has been found, using sodium carboxylates in the presence of cuprous chloride.

As part of an on-going synthetic project in our laboratory, we required an effective way to prepare the monoprotected cis-2-cyclohexene-1,4-diol, 1 , in multigram quantities. Yamada, et al. recently prepared several monoesters of general formula 1 , by reaction of cis-2-cyclohexene-1,4-diol with one equivalent of acyl chloride. ${ }^{1}$ In most cases, chromatographic purification is necessary to separate the monoester from the diester.

Our previous experience ${ }^{2}$ with the regiospecific addition of organocyanocuprates to cyclic vinyl epoxides prompted us to examine the use of cuprous ion in the synthesis of monoprotected cis-1,4-diols. More specifically, we sought a simple method to effectively add carboxylic acid salts in a syn-1,4 fashion across the monoepoxide of 1,3-cyclohexadiene. We discovered that the sodium salts of carboxylic acids would rcact with the epoxide, in the presence of one equivalent of $\mathrm{CuCl}$, to give the desired 1,4-cis adducts with very high regio- and stereoselectivity (see scheme 1). The hydroxyesters can then be distilled or used without further purification. As shown in Table I, the monoepoxide of cyclopentadiene also reacts in a similar fashion.

\section{Scheme 1}

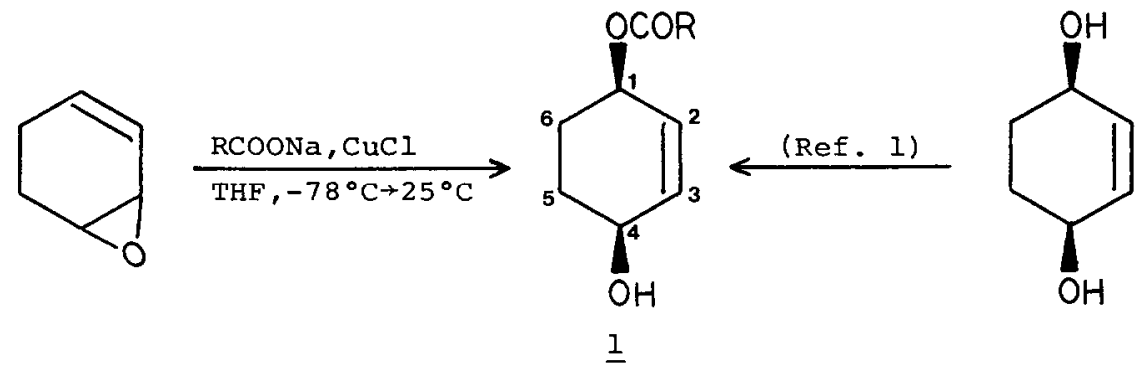




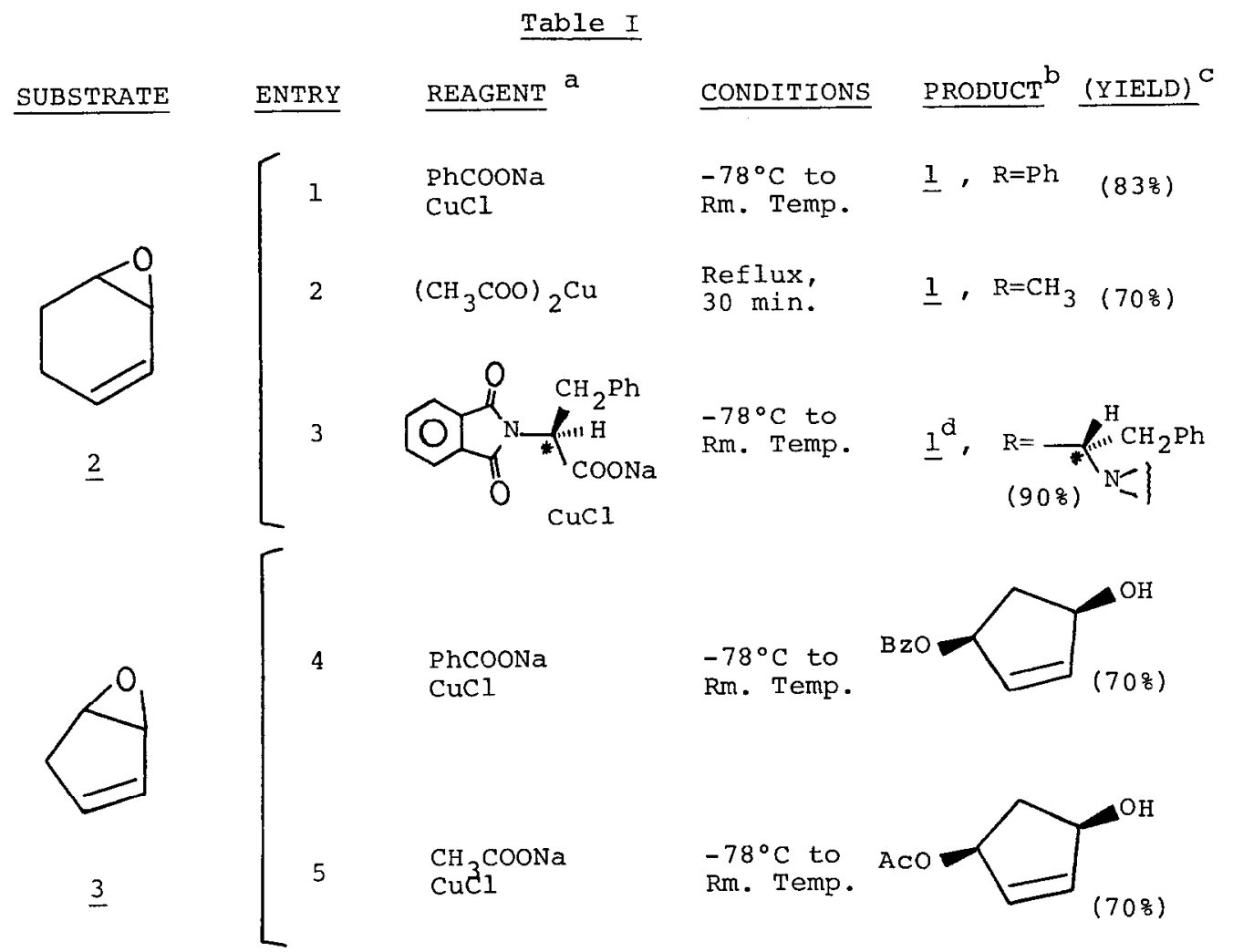

$a_{A 11}$ reactions were carried out in ary THF, at concentrations below $0.1 \mathrm{M}$.

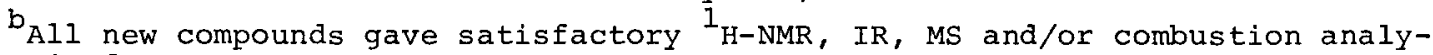
sis data.

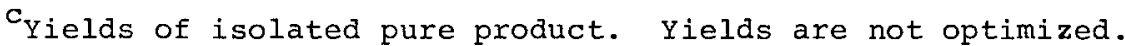

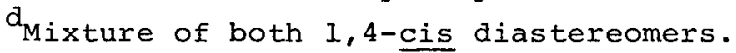

The 1,4 adducts obtained were shown to possess cis-stereochemistry by comparison of their ${ }^{1} \mathrm{H}$-NMR spectra with the spectroscopic information published by Yamada for these compounds. ${ }^{1}$ For a chemical verification of the stereochemistry of the adducts, we synthesized the corresponding trans isomers by a modification of a Sharpless procedure. ${ }^{3}$ The cis and trans isomers were clearly different in their $360 \mathrm{MHz}{ }^{1} \mathrm{H-NMR}$ and ${ }^{13} \mathrm{C}-\mathrm{NMR}$ spectra. While cis-benzoate $\underline{I}(R=P h)$ exhibited vicinal coupling constants $J_{12}$ and $\mathrm{J}_{34}$ in the $4 \mathrm{~Hz}$ range, the corresponding coupling constants in the trans isomer were in the $2-2.5 \mathrm{~Hz}$ range. In regard to ${ }^{13} \mathrm{C}-\mathrm{NMR}$, the main difference between the cis and trans isomers may be found in carbon-1 (see scheme 1) Thus, $\mathrm{C}-1$ in cis-acetate $\underline{1}\left(\mathrm{R}=\mathrm{CH}_{3}\right)$ was 1 ppm upfield from the trans isomer $(67.357$ and $68.386 \mathrm{ppm}$, respectively). 
While this copper-mediated transformation has no clear precedent in the literature, the palladium catalyzed addition of carbon nucleophiles to vinyl epoxides is well known to give cis-1,4-products. ${ }^{4-6}$ The 1,4-addition of heteroatoms to vinyl epoxides is not extensively documented, with the exception of one case involving a nitrogen nucleophile. ${ }^{5}$ Recently, Bäckvall, et al., reported a novel process that allows the selective transformation of 1,3-dienes into cis or trans 1,4-diacetoxy-2-alkenes using a Pd(0) catalyst. 7

At this staye of our study, we believe that this $\mathrm{Cu}(\mathrm{I})$ mediated reaction could involve the initial formation of a copper carboxylate. It is well established that $C u(I)$ carboxylates react with alkyl and vinyl halides ${ }^{8}$ via an oxidative addition of copper to the carbon-halogen bond followed by displacement of copper by a carboxylate anion. In the present reaction, we envisage a copper(I) carboxylate species that is capable of both complexation with the epoxide oxygen atom and intramolecular delivery of the carboxylate anion. Such a transition state would require an s-cis orientation of the double bond and the epoxide.

In this context, we have found epoxides $\underline{4}$ and $\underline{5}$ to be completely unreactive under the conditions developed for epoxides $\underline{2}$ and $\underline{3}$, even when the reaction was kept at reflux for hours. This can be rationalized by the transoid nature of the allylic epoxide system of $\underline{4}$ and $\underline{5}$, where the terminal position of the double bond is effectively too distant from the complexing copper for reaction to occur. Trans-substituted epoxide $\underline{6}$, whose s-cis conformation must be less sterically strained than that of epoxide $\underline{5}$, was unreactive at low temperature, but after refluxing in THF for seven days with $\mathrm{PhCOONa/CuCl}$ a mixture of trans-1,4-adduct and 1,2-adducts was obtained. ${ }^{9}$ Thus, at the present time, our synthetic method seems to be limited to cyclic vinylic epoxides.

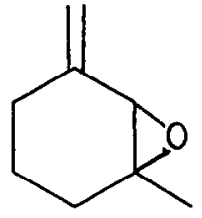

$\underline{4}$

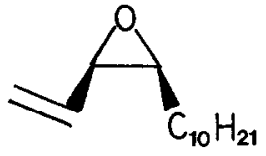

$\underline{5}$

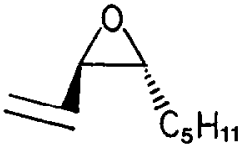

$\underline{6}$

The reaction conditions used for the opening of the cyclic vinyl epoxides are very mild and should not interfere with numerous functional groups. A typical experimental procedure is as follows: Anhydrous sodium benzoate $(21.6 \mathrm{~g}, 0.15 \mathrm{~mole})$ and cuprous chloride $(14.85 \mathrm{~g}, 0.15$ mole) were added to 
$1.0 \mathrm{~L}$ of dry THF and stirred under nitrogen for 30 minutes at room temperature. After cooling the resulting fine suspension to $-78^{\circ} \mathrm{C}$, a solution of 1,3 -cyclohexadiene monoepoxide $(7.25 \mathrm{~g}, 0.075 \mathrm{~mole})$ in $10 \mathrm{ml}$ of $\mathrm{THF}$ was added via syringe. Stirring was continued at $-78^{\circ} \mathrm{C}$ for $2 \mathrm{~h}$ and then at room temperature overnight. The reaction mixture was quenched with $500 \mathrm{~mL}$ of a saturated $\mathrm{NH}_{4} \mathrm{Cl}$ solution. After filtration through Celite, the aqueous layer was extracted with ethyl acetate $(5 \times 75 \mathrm{ml})$, and the combined organic solutions were washed sequentially with $10 \% \mathrm{NH}_{4} \mathrm{OH}, \mathrm{NaHCO}_{3}$ and NaCl solutions. Drying over $\mathrm{MgSO}_{4}$ and concentration in vacuo provided $13.90 \mathrm{~g}$ (84\% yield) of crude hydroxyester 1 ( $\mathrm{R}=\mathrm{Ph}), \mathrm{bp} 124-128^{\circ} \mathrm{C}(1 \mathrm{~mm} \mathrm{Hg})$.

Acknowledgments. This research was supported in part by a grant from the National Cancer Institute (PHS), CA 22237. We also wish to acknowledge the National Science Foundation for support of a Brüker $360 \mathrm{MHz}$ NMR spectrometer.

\section{$\underline{\text { References }}$}

1. M. Nara, S. Terashima and S. Yamada, Tetrahedron, 36, 3161 (1980).

2. J. P. Marino and D. M. Floyd, Tetrahedron Lett., 675 (1979); J. P. Marino

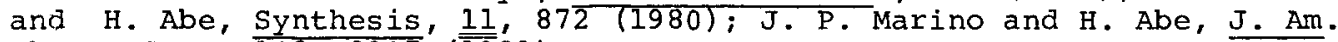
Chem. Soc., $\underline{\underline{103}, 2907}$ (1981).

3. K. B. Sharpless and R. F. Lauer, J. Am. Chem. Soc., 95, 2697 (1973). our modification involves the following sequence:

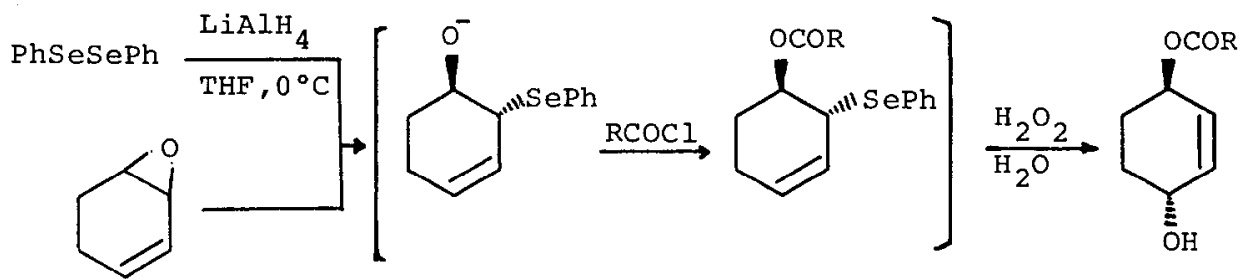

4. B. M. Trost and G. Molander, J. Am. Chem. Soc., 103, 5969 (1981).

5. J. Tsuji, H. Kataoka and Y. Kobayashi, Tetrahedron Lett., 2575 (1981).

6. B. M. Trost and T. Runge, J. Am. Chem. Soc., 103, 7550 (1981).

7. J. Bäckvall, R. Nordberg and J. Nystrom, Tetrahedron Lett., 1617 (1982).

8. A. H. Lewin and N. Goldberg, Tetrahedron Lett., 491 (1972).

9. The 1,2-adduct, obtained in 53\% yield, consisted of a mixture of the product from direct allylic opening of the epoxide and the secondary product derived from 1,2-acyl transfer. The 1,4-adduct (348 yield) was all trans from its $360 \mathrm{MHz}{ }_{\mathrm{H}-\mathrm{NMR}}$ and no cis-alkene could be detected.

(Received in USA 7 June 1982) 